\title{
PERIPHERAL ARTERIOGRAPHY AND AORTOGRAPHY
}

\author{
By Robert E. Steiner, M.B., Ch.B., D.M.R., F.F.R., and David Messent, M.B., F.R.C.S. \\ Departments of Radiology and Surgery, Hammersmith Hospital (Postgraduate Medical School of London)
}

\section{Introduction}

The radiological demonstration of peripheral blood vessels in the human cadaver was first achieved by Haschek in 1896 . In 1923 Berberich and Hirsch carried out the first in vivo arteriogram, but it was not until the introduction of safe radioopaque compounds, such as diodone, that the method came into universal practice. Today there is hardly a vascular system in the human body which has not been explored by arteriography. In this paper it is proposed to limit the discussion to arteriography of the limbs and to aortography.

\section{Peripheral Arteriography}

Clinical examination will usually provide adequate information for the management of the patient suffering from peripheral vascular disorders. If, however, additional knowledge is required, arteriography is the best ancillary method of investigation. It will demonstrate with accuracy the presence of organic disease, the level and extent of arterial blocks, the patency of the vessels distal to the block and the efficiency of the collateral circulation.

\section{Indications for Peripheral Arteriography}

(a) In obliterative arterial disease with the object of establishing and confirming the presence of early disease; localizing arterial thrombosis to aid direct surgical treatment, such as arterial grafting or end-arterectomy; and providing a guide to the level of amputation.

(b) To demonstrate aneurysms and arteriovenous fistulae.

(c) To aid the diagnosis of vascular tumours such as bone sarcomas and haemangiomas.

Peripheral arteriography should be carried out under general anaesthesia, which avoids movement due to the pain produced by the intra-arterial injection of diodone. Percutaneous injection is the method of choice since exposure of the artery turns a relatively simple procedure into a formal operation. If percutaneous injection has failed or the artery cannot be defined by palpation, dis- section will become necessary and is safer than repeated attempts at percutaneous arterial puncture.

\section{Contrast Medium}

Fifty per cent. diodone is the most satisfactory medium for peripheral arteriography. If injected into the soft tissues, it will give rise to local discomfort, but it is absorbed and excreted without residual effects. For arteriography of the lower limb $20 \mathrm{cc}$. of contrast medium are required, while ro to $15 \mathrm{cc}$. are adequate for the upper limb. In some centres thorotrast is used; its intra arterial injection is painless but the substance is not excreted. In view of its radioactive propertie and the fact that it is permanently fixed in the reticulo-endothelial system of the body, its after? effects are considered dangerous by some workers (Taft, I937).

Prior to the use of diodone, the patient should be tested for sensitivity to this drug by the injection of $2 \mathrm{cc}$. intravenously. Coughing, increase in pulse rate, occurrence of a rash and salivation are the signs of sensitivity and desensitization must be carried out before arteriography can be performed.

\section{Arteriography of the Lower Limb Injection Technique}

Through a No. I $8 \mathrm{G}$ short bevel needle $20 \mathrm{cc}$. of 50 per cent. diodone solution are injected into the femoral artery just below the inguinal ligament. The needle point must be well within the lumen of the artery and, if possible, should be advanced down the vessel for a short distance to avoid intramural injection or a leak at the point of puncture. The injection is carried out as rapidly as possible whilst the artery is compressed above the point of injection by an assistant until the second radiograph is exposed, thus avoiding too rapid dilution of the contrast medium. Alternatively the flow of contrast medium may be directed against the arterial stream (Lindbom, 1950). Following the withdrawal of the needle, compression of the 


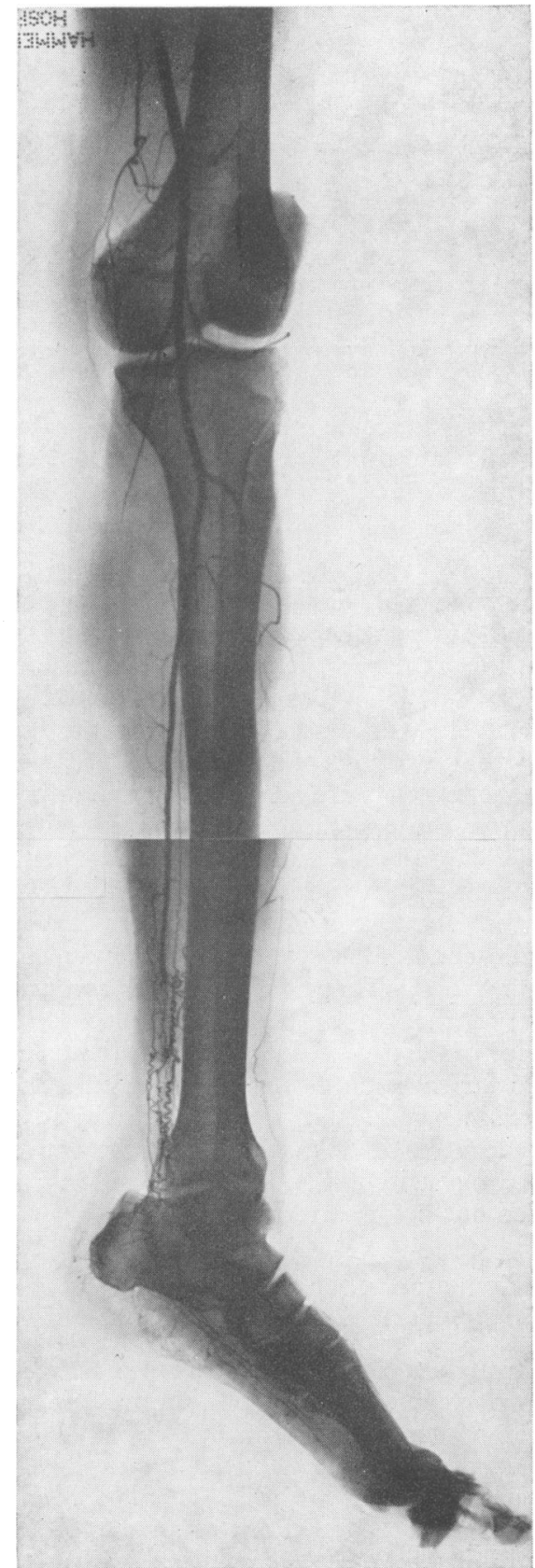

Fig. I.-Arteriogram of the right lower limb-a male patient aged $3 \mathrm{I}$. The tibial arteries are thrombosed. The circulation of the foot is inadequate-carried entirely through tortuous collateral vessels. This is an example of the peripheral type of arterial disease - 'thrombo-angiitis obliterans.' The femoral and popliteal arteries are perfectly normal.

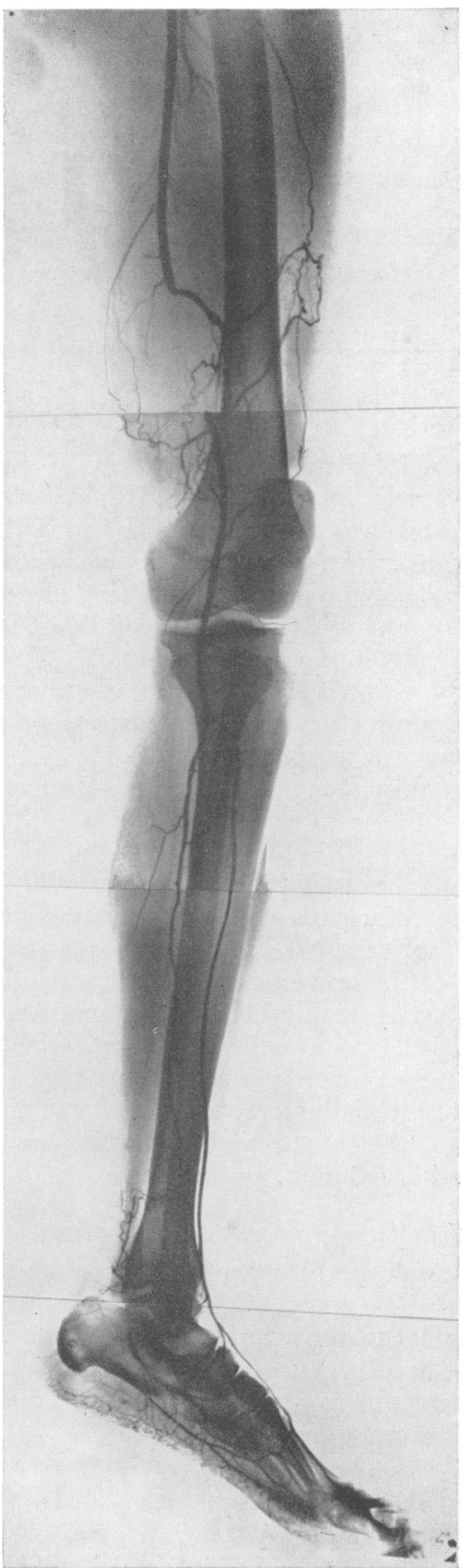

FIG. 2.-Male patient aged 5I (left leg). The arteriogram demonstrates thrombosis of the femoral artery at the level of Hunter's canal with good $N$ collateral circulation. The posterior tibial is also $\sigma$ thrombosed in the calf, a short segment just above $<$ the ankle being patent. Atheromatous changes are seen in the tibial vessels distal to the bifurcation. $\Phi$ The circulation of the foot appears to be very? adequate. An example of 'degenerative arterial $\square$ disease.' 
artery over the puncture site is maintained for a while to prevent haematoma formation.

\section{Radiographic Technique}

The limb is externally rotated and placed on a cassette tunnel which extends from groin to toe. In this position serial antero-posterior radiographs are taken at rapid intervals. Three $15 / 12$ films and one 12/10 film will cover the limb and allow for adequate overlap. Cassettes are placed in succession beneath the thigh, knee, calf and foot, the tube being re-centred after each exposure. Rapid cassette changing is essential; the whole procedure should not exceed 20 seconds. By this method the main arterial tree of the whole limb can be adequately demonstrated.

\section{Arteriography of the Upper Limb}

Demonstration of the axillary and brachial arteries is difficult without formal exposure, however direct puncture of the third part of the subclavian and of the axillary artery is possible. The arteries of the forearm and hand are best visualized by injection of the brachial artery above the elbow joint. A short bevel No. $19 \mathrm{G}$ needle is used and injection is carried out by an assistant through the side piece of a three-way tap, the operator holding the needle steady in the arterial lumen. Blood flow in the limb is controlled by a sphygmo-manometer cuff which is placed on the arm above the point of injection. The pressure in the cuff must be raised to above systolic pressure after the artery has been successfully punctured and before the injection is commenced. The first film is taken after completion of injection. The pressure in the sphygmo-manometer cuff is then lowered to diastolic levels for one or two pulse beats and at once raised again to systolic levels. This procedure is carried out between each radiographic exposure.

\section{Radiographic Technique}

The hand and forearm are spread out in the supine position on a cassette tunnel, with the fingers extended. Multiple exposures are made at intervals of two to three seconds, the arterial blood flow being controlled as previously described. Six exposures are considered adequate. A 12/10 film will cover the hand and lower forearm; if the upper forearm is to be included, a $15 / 12$ cassette is necessary.

\section{Interpretation}

\section{Normal Appearances}

All major arteries distal to the point of injection are outlined as far down as the wrist or ankle. The vessel walls are smooth and parallel, diminishing in calibre towards the periphery. The main branches to skin and muscle fill with contrast medium only for a short distance. In the hand the palmar arch and digital arteries are clearly outlined but in the foot detail is poor beyond the dorsalis pedis and plantars. There is evidence of early venous filling in both hands and feet. No collateral vessels are seen. The circulation, as judged by arteriography, can only be assessed qualitatively and not quantitatively.

Appearances in Obliterative Arterial Disease (Figs. I and 2)

In assessing the arteriogram the following points must be taken into account:

I. Presence of irregularities of the vessel walls and narrowing of the origins of main branches, indicating atheroma.

2. The site and extent of an arterial block.

3. Presence of collateral vessels, their number, tortuosity and calibre.

4. Refilling of the main arteries beyond the blocks and the speed at which this takes place. Failure to demonstrate refilling may be due to excessive delay in the circulation.

5. The delay or absence of venous filling.

We consider that the early signs of obliterative arterial disease are the appearance of slight irregularites in the vessel walls, narrowing of the origins of main branches and the development of collateral vessels which appear as tortuous arterial channels in abnormal anatomical positions.

\section{Arterio-venous Fistulae (Fig. 3)}

Congenital arterio-venous fistulae which are commonly multiple and of great complexity are often impossible to demonstrate. In acquired arterio-venous fistulae the site and extent of the anastomoses can readily be shown.

\section{Aneurysms}

The site and size of the aneurysmal sac can be demonstrated and the efficiency of any collateral vessels assessed.

\section{Complications}

The most serious complication is the precipitation of arterial thrombosis either at the site of injection or distal to it, and this is the main reason why arteriography should not be carried out as a routine investigation. The incidence of this complication is rare; it has occurred only once in our series of over 250 peripheral arteriograms. Of minor importance are haematoma formation and peri-arterial injection, neither of which cause any permanent damage. Other complications which have been reported, but of which we have no experience, are the dislodgement of atheromatous 


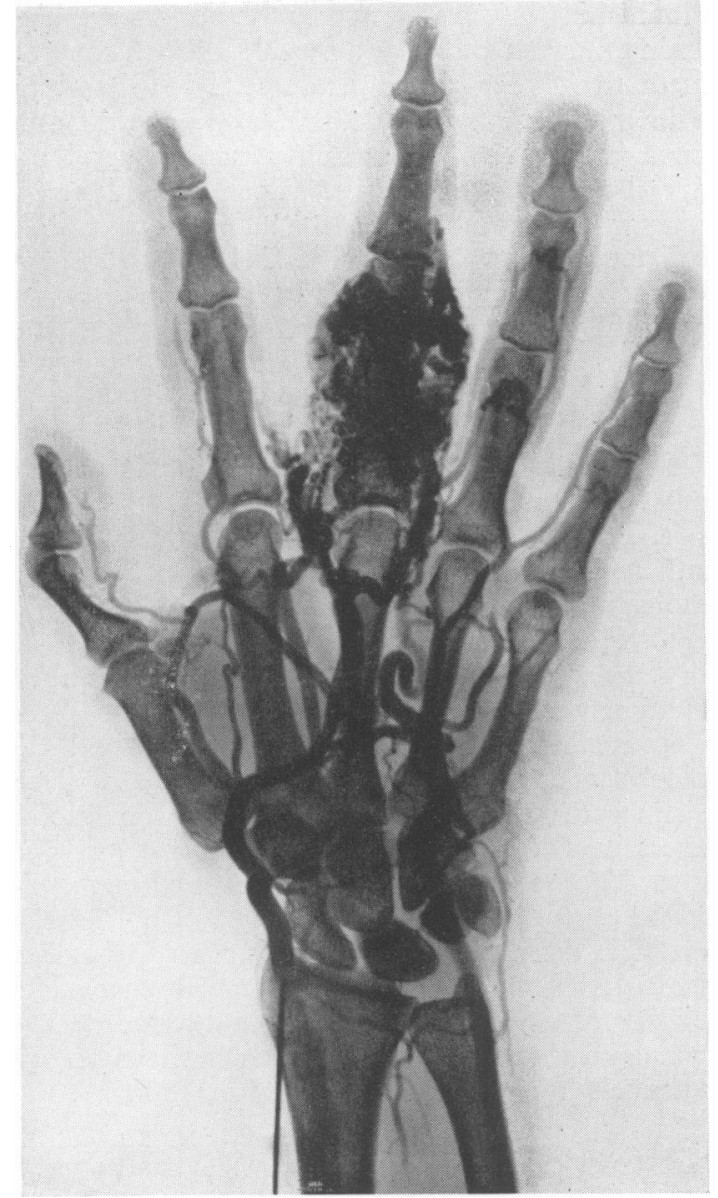

Fig. 3.-Congenital arterio-venous fistula of the hand of a girl aged 16.

plaques at the site of injection and also the development of arterio-venous fistulae.

\section{AORTOGRAPHY}

The first radiographic demonstration of the human abdominal aorta with the aid of contrast media was accomplished by the Portuguese urologist, Dos Santos, and his colleagues, in 1929 by direct injection into the aorta in the lumbar region. Since then the scope has greatly widened to include the thoracic aorta and the great vessels of the neck. For the purpose of this discussion it is convenient to consider thoracic and abdominal aortography separately.

\section{Thoracic Aortography Indications}

I. Coarctation of the aorta where venous angiography has failed to give adequate information.

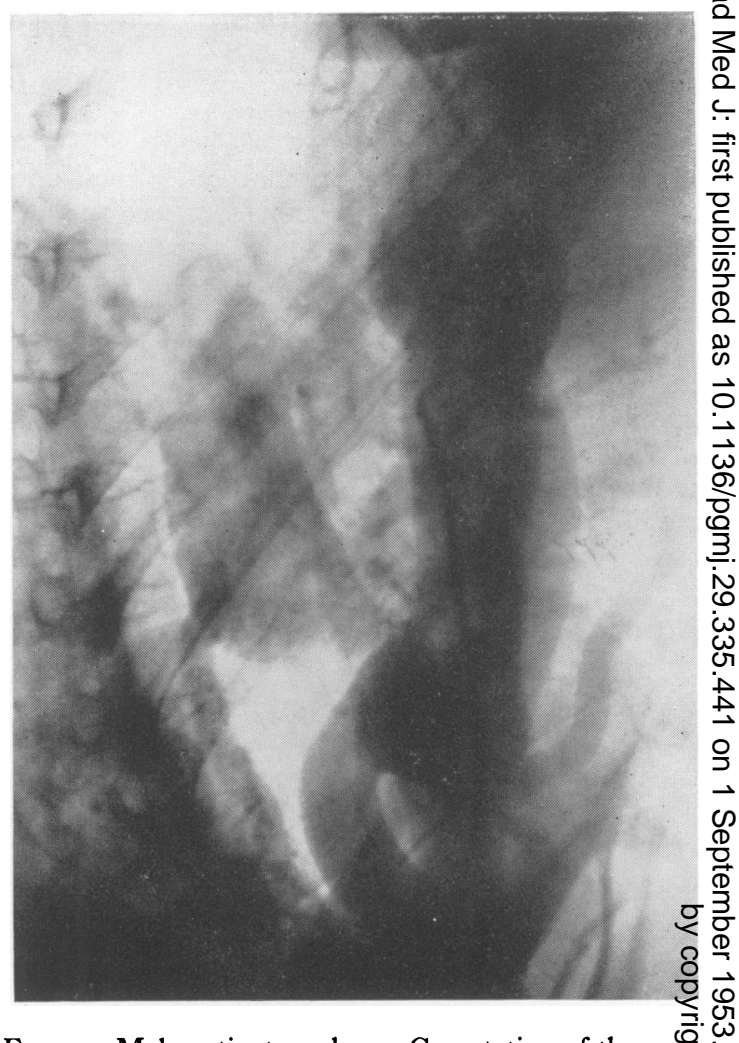

FIG. 4.-Male patient aged 23. Coarctation of the aor demonstrated by retrograde aortography. By kind permission of the editor of the British Fournal of Radiology.

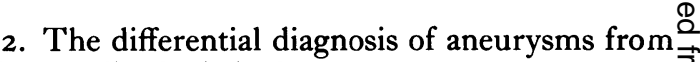
other mediastinal shadows.

3. The localization of arterio-venous fistulae.

\section{Technique}

Direct puncture of the thoracic aorta through $\frac{}{3}$ the second left intercostal space has been carried-: out by Radner (1945) and Campo and Hoyos 3 (1948), but this method has not found general acceptance due to its potential danger.

Jonsson (1945) described percutaneous puncture of the carotid with a cannula. The axillary and brachial arteries were first used by Castellanos and $D$ Pereiros (1939), but not until Broden et al. (19481949) described retrograde catheterization of the $N$ radial artery did the method become firmly 0 established and the most commonly used today.

The radial artery is exposed near its origin in the antecubital fossa under general anaesthesia; a No.? 8 or 9 radio-opaque U.S.A. gauge cardiac catheter is threaded into the radial artery under fluoro- $\mathbb{D}$ scopic control and is directed into its required ${ }^{+}$ position in the thoracic aorta. The right radial $\frac{0}{\circ}$ 


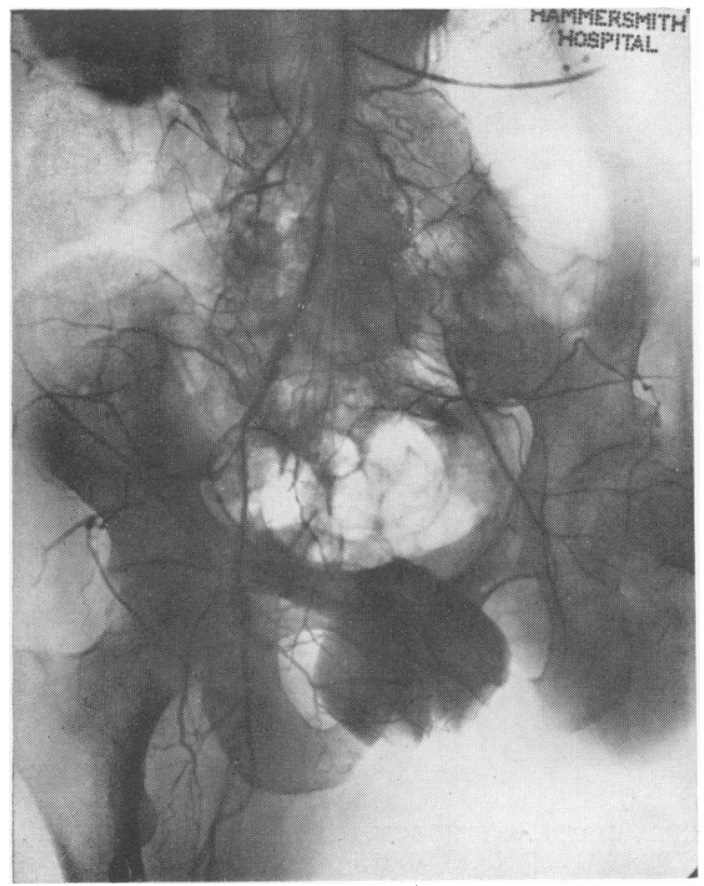

FIG. 5.-Abdominal aortogram by direct puncture; a male patient aged 47 . There is complete thrombosis of the left common iliac artery. In the right iliac artery marked atheroma is demonstrated with almost complete obliteration of a short segment. (The left leg had been amputated for gangrene of the foot.)

artery should be used since, like Broden et al. (1948) we have found that the catheter passed up the left artery will more readily enter the descending aorta. We have used this latter approach for aortography. of the descending and abdominal aorta. Local arterial spasm may give rise to difficulties when passing the cardiac catheter but rapid passage of the catheter helps to overcome this complication as does the local application of heat or $2 \frac{1}{2}$ per cent. papaverin. The exact details of the technique are described by Broden et al. (1949). The contrast medium of choice is 70 per cent. diodone; $50 \mathrm{ml}$. are injected with the aid of a pressure pump as rapidly as possible (three to five seconds).

\section{Radiographic Technique}

A serial cassette changer, either manually or mechanically operated, is essential in the investigation of coarctation or arterio-venous fistulae, and it is preferable to carry out the investigation in two planes. For aneurysms and obliterative arterial disease these elaborate radiographic techniques are not essential and one or two films exposed at the right moment can give the necessary information.

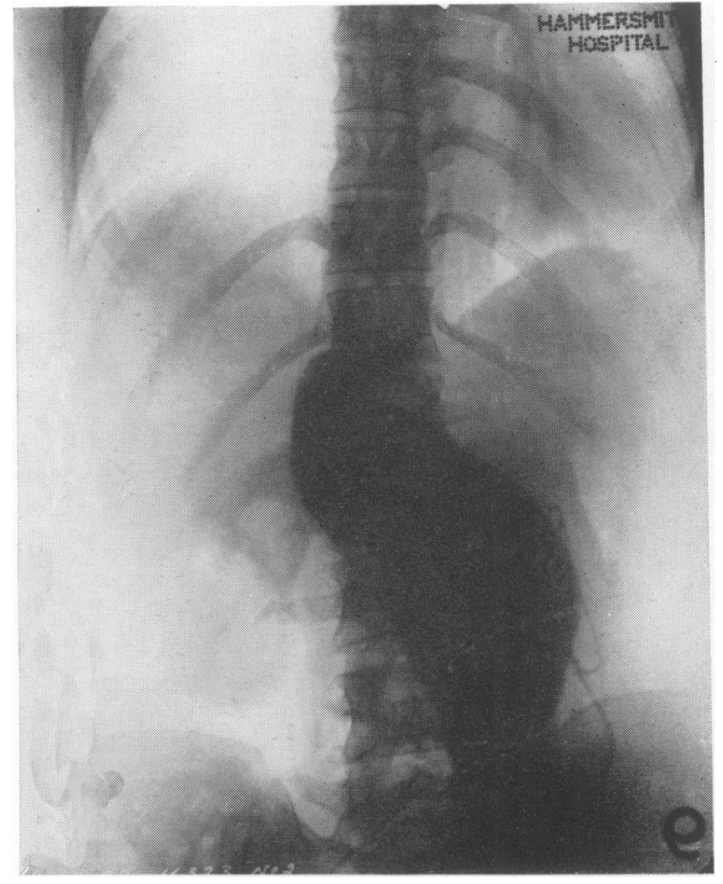

FIG. 6.- Female patient aged 36. Aneurysm of the $\frac{\sigma}{0}$ abdominal aorta demonstrated by retrograde aortography through right femoral artery.

\section{Interpretation}

Coarctation of the aorta (Fig. 4). The aortogram will demonstrate the site and extent of the lesion accurately, the relationship of the subclavian artery, the state of the aorta above and below the coarctation and the presence of any other anomalies as, for example, persistent ductus or any aneurysm. A decision as regards operability and the type of operation performed will largely depend on the radiographic findings.

\section{Abdominal Aortography \\ Indications}

I. Obliterative arterial disease affecting the aorta and iliac vessels.

2. Aneurysms and arterio-venous fistulae.

3. Certain urological conditions.

4. Placenta praevia.

\section{Technique}

Direct puncture of the abdominal aorta. The basic principles of this technique were established by Dos Santos in 1929. Since then many modifications have been described and the literature has recently been reviewed by Detterling (1952).

'The patient lies prone on the X-ray table and the aorta is approached from behind, using a $16 \mathrm{G}$ 
$\mathrm{x} 6$ in. needle. The needle is inserted a hand's breadth from the midline on the left side opposite the first lumbar vertebra. It is directed upwards and medially beneath the twelfth rib towards the body of the twelfth dorsal vertebra. Once it is felt to impinge on the vertebral body it is withdrawn slightly and then further advanced in a more vertical direction to slide past the edge of the bone. The needle point is felt to enter the aorta after it has been advanced another $2 \mathrm{~cm}$., and blood will be seen to stream back readily into the attached syringe. The needle should then be advanced another $\frac{1}{2} \mathrm{~cm}$. to ensure that the needle point is well within the aortic lumen. With the aid of a pressure pump $50 \mathrm{ml}$. of 70 per cent. diodone are injected in three to five seconds. In all three to four films are exposed, the first radiograph being taken after two-thirds of the contrast medium has been injected. A cassette tunnel with a fixed grid, manually or mechanically operated cassette changers or potter bucky films will give satisfactory results. Some workers advise occlusion of the lower limb circulation except when investigating obliterative arterial disease.

Retrograde aortography. The aorta can be approached from the left forearm as mentioned above or via the femoral artery and its branches below Pouparts ligament (Castellanos and Pereiras, 1940). The method is very similar to retrograde thoracic aortography. In investigations of aneurysms of the aorta, the retrograde method is the one of choice.

\section{Interpretation}

In obliterative arterial disease, the points to note have already been discussed under the section on peripheral arteriography (Fig. 5). It is essential to have accurate anatomical information if re- constructive surgery is to be carried out. The same points apply to the investigation of aneurysms (Fig. 6) and arterio-venous fistulae. In certain urological conditions aortography is of value to demonstrate abnormalities of renal vessels, the displacement of vessels by space-occupying lesions and the demonstration of abnormal vessels in vascular tumours.

\section{Complications of Aortography}

Peri-vascular injection will give rise to temporary pain but in itself is harmless.

Injury to the aortic wall and haematoma formation may also ensue but in practice little trouble has occurred from these complications.

Precipitation of thrombosis in distal vessels is a danger, particularly in obliterative arterial disease.

In thoracic aortography, damage to the cerebral arteries and coronary ischaemia have been noted if too high a concentration of contrast medium reaches these vessels.

\section{BIBLIOGRAPHY}

BERBERICH, J., and HIRSCH, S. (1923), Klin. Wschr., 2, 2226. BRODEN, B., HANSON, H. E., and KARNELL, J. (1948), Acta Radiol. Stockh., 29, 181.

BRODEN, B., JONSSON, G., and KARNELL, J. (1949), Ibid 32, 498.

CASTELLANOS, A., and PEREIRAS, R. (1940), Rev. cubang cardiol., 2, 187 .

CASTELlANOS, A., PEREIRAS, R., and GARCIA, A. (193\% $\overrightarrow{0}$ F. Pediat. Santa Clara. (quoted by Castellanos and Pereiras, of 1940).

DETERLING, R. A. (1952), Surgery, 31, 88.

DOS SANTOS, R., LAMAS, C., and PEREIRA CALDAS, ' $\mathrm{J}$. (1929), Bull. Soc. nat. Chir., 55, 587.

HASCHEK, E., and LINDENTHAL, C. T. (1896), Wien. klin Wschr., 9, 63.

JONSSON, G. (1949), Acta Radiol. Stockh., 31, 376.

LINDBOM, A. (1950), Acta Radiol. Stockh. Suppl., 80, 10.

MENESES HOYOS, J., and GOMEZ DEL CAMPO, C. (1948), Radiology, 50, 211 .

RADNER, S. (1945), Acta Radiol. Stockh., 26, 497.

TAFT, R. B. (1937), f. Amer. med. Ass., 108, 1779.

\section{RUTHIN CASTLE, NORTH WALES}

A Clinic for the diagnosis and treatment of Internal Diseases (except Mental or Infectious Diseases). The Clinic is provided with a staff of doctors, technicians and nurses.

The surroundings are beautiful. The climate is mild. There is central heating throughout. The annual rainfall is 30.5 inches, that is, less than the average for England.

The Fees are inclusive and vary according to the room occupied.

For particulars apply to THE SECRETARY, Ruthin Castle, North Wales. 